\title{
PATÓgENO MÁS COMÚN, SENSIBILIDAD ANTIBIÓTICA Y FACTORES DE RIESGO ASOCIADOS A SEPSIS NEONATAL EN EL HOSPITAL TEODORO MALDONADO CARBO EN EL PERIODO 2013-2014
}

MOST COMMON PATHOGEN, ANTIBIOTIC SENSITIVITY, AND RISK FACTORS ASSOCIATED WITH NEONATAL SEPSIS IN TEODORO MALDONADO CARBO HOSPITAL IN 2013-2014

PATÓGENO MAIS COMUM, SENSBBLLIDADE ANTIBIÓTICA EFATORES DE RISCO ASSOCIADOS Ȧ SEPSENEONATAL NO HOSPTTAL TEODORO MALDONADO CARBO NO PERÍODO 2013-2014

\author{
NADYA BEJARANO TORRES, KATHERINE ESTAY PERALTA \\ Universidad Católica de Santiago de Guayaquil; Guayaquil, Ecuador
}

Resumen

Objetivo: determinar los microorganismos más frecuentes aislados en neonatos con diagnóstico de sepsis, sus patrones de sensibilidad y resistencia antibiótica, asi como la identificación del tipo de sepsis neonatal más prevalente y los factores de riesgo más comunes para cada uno. Planteándose la hipótesis de que Stafilococus Epidermidis es el patógeno más común aislado en los casos de sepsis neonatal. Metodologia: se diseñó un estudio observacional, descriptivo con datos retrospectivos, que incluyó neonatos con sospecha de sepsis en la unidad materno infantil del hospital Teodoro Maldonado Carbo, en el periodo de enero de 2013 a diciembre de 2014. Resultados: de 163 pacientes con sospecha de sepsis, el 58\% de la muestra no se pudo comprobar el diagnóstico. El tipo de sepsis prevalente fue de origen tardío con el $59,42 \%$, siendo la corioamnionitis el único factor obstétrico con riesgo significativo para sepsis temprana ( $\mathrm{p}=0,041$; IC 95\%) y la condición de pretermino extremo $<32$ sg para sepsis tardía ( $\mathrm{p}=0,017$; IC 95\%). Los estafilococos coagulasa negativo fueron los más frecuentes. S. epidermidis (33.8\%) en ambos tipos de sepsis. Conclusión: el control de los factores de riesgo infecciosos de origen obstétrico, reduce la incidencia de sepsis neonatal temprana; sin embargo surgen de manera concomitante las infecciones por microorganismos nosocomiales, sobre todo en recién nacidos pre término. Es necesario el cumplimiento estricto de los protocolos de higiene y seguridad del personal e insumos médicos y el uso mesurado de antibioticoterapia empírica ante la sospecha de sepsis neonatal.

PALABRAS CLAVE: sepsis neonatal, factores de riesgo, unidades de cuidado intensivo neonatal,corioamnionitis,pretérmino, infección hospitalaria.

Abstract

Objective: determine the most frequent isolated microorganisms in neonates diagnosed with sepsis, their patterns of sensitivity and antibiotic resistance, as well as the identification of the most prevalent type of neonatal sepsis and most common risk factors for each one. Hypothesis, Staphylococcus epidermidis is the most common isolated pathogen in neonatal sepsis. Methodology: our study design was observational, descriptive with retrospective data, which included neonates with a suspected sepsis in the maternal child unit of the Teodoro Maldonado Carbo hospital, from January 2013 to December 2014. Results: from 163 patients with suspected sepsis, $58 \%$ of the participants could not confirm the diagnosis. Late origin sepsis was the most prevalent Type of sepsis in our study, with $59.42 \%$ of the participants, chorioamnionitis was the only obstetric factor with significant risk for early sepsis ( $\mathrm{p}=0.041 ; 95 \% \mathrm{Cl})$ and the extremely preterm birth conditions (<32 gestational age) for late sepsis. Coagulase-negative staphylococci were the most frequent. s. epidermidis (33.8\%) present in both types of sepsis. Conclusion: the control of infectious risk factors in obstetric patients reduces the incidence of early neonatal sepsis. However, nosocomial microorganism infections arise concomitantly, especially in preterm birth patients. Strict fulfillment with hygiene and safety protocols for staff and medical supplies are necessary and the measured use of empirical antibiotic therapy for the presence of suspected neonatal sepsis.

KEYWORDS: neonatal sepsis, risk factors, intensive case units neonatal, chorioamnionitis, premature, cross infection.

Resumo

Objetivo: determinar os microrganismos mais frequentes isolados em neonatos diagnosticados com sepse, seus padrões de sensibilidade e resistência a antibióticos, bem como identificar o tipo de sepse neonatal mais prevalente e os fatores de risco mais comuns para cada um. Afirmando a hipótese de que 0 Stafilococus Epidermidis é o patógeno mais comum isolado nos casos de sepse neonatal. Metodologia: estudo observacional descritivo, com dados retrospectivos, que incluiu neonatos com suspeita de sepse na unidade materno-infantil do hospital Teodoro Maldonado Carbo, no período de janeiro de 2013 a dezembro de 2014. Resultados: de 163 pacientes com suspeita da sepse, 58\% da amostra não pôde verificar o diagnóstico. 0 tipo prevalente de sepse foi de origem tardia com 59,42\%, sendo a corioamnionite o único fator obstétrico com risco significativo de sepse precoce $(p=0,041$; IC95\%) e condição pré-termo extrema $<32 \mathrm{sg}$ para sepse tardia ( $p=0,017$; IC95\%). Estafilococos coagulase-negativos foram os mais frequentes. S. epidermidis $(33,8 \%)$ em ambos os tipos de sepse. Conclusão: 0 controle de fatores de risco infecciosos de origem obstétrica reduz a incidência de sepse neonatal precoce; no entanto, infecções por microrganismos nosocomiais surgem concomitantemente, especialmente em prematuros. 0 cumprimento estrito dos protocolos de higiene e segurança para pessoal e suprimentos médicos e o uso medido da antibioticoterapia empírica são necessários na presença de suspeita de sepse neonatal. PALAVRAS-CHAVE: sepse neonatal, fatores de risco, unidades de terapia intensiva neonatal, corioamnionite, prematuro, infecção hospitalar. 


\section{INTRODUCCIÓN}

La sepsis neonatal se define como un cuadro clínico en el que se desarrolla el síndrome de respuesta inflamatoria sistémica (SRIS) o fetal (SRIF), con la manifestación de dos o más signos clínicos, junto a los que deben encontrarse alteraciones de laboratorio, así como aislamiento de algún patógeno en cultivo de sangre dentro de los primeros 28 días de vida del ser humano. ${ }^{1,2,3,4}$ A su vez, se divide en sepsis neonatal temprana, dentro de las primeras 72 horas de vida, con transmisión de tipo vertical, factores de riesgo maternos y fetales; y sepsis neonatal tardía que muestra signos y /o síntomas de infección posterior a las primeras 72 horas de vida neonatal, con transmisión de tipo horizontal, de la comunidad o intrahospitalaria, e intervienen factores de riesgo ambientales. . $^{2,5,6,7}$

A nivel mundial en el 2013 se reportaron alrededor de 2,8 millones de muertes neonatales (44\% del total de muertes en menores 5 años (de este, el $36 \%$ el primer día de vida, el $37 \%$ en etapa neonatal temprana y el $27 \%$ en etapa neonatal tardía). ${ }^{8,6}$ Se ha estimado que a nivel mundial más de 1.4 millones de muertes neonatales son resultado de infecciones dentro de las cuales se incluye a la sepsis neonatal. ${ }^{9}$

La sepsis corresponde al 15,6\% responsable de muerte neonatal, entre esos el $47,6 \%$ en etapa temprana. ${ }^{8,10}$ Según diversos estudios el $23 \%$ de los ingresos a UCIN (Unidad de Cuidados Intensivos) presentan sepsis, $4 \%$ sepsis grave y $2 \%$ shock séptico; además con un aumento de la mortalidad observada al progresar en gravedad hasta un $55 \% .{ }^{11}$ En el Ecuador según el INEC (Instituto Nacional de Estadísticas y Sensos) en el año 2013 se dieron 2928 muertes en menores de 1 año de edad, de las cuales 1599 fueron en etapas neonatales (1099 en etapa neonatal temprana y 500 neonatal tardía), de éstas, 804 se dieron en la región Costa y 566 en la Provincia del Guayas. ${ }^{12}$

Los autores se plantean el siguiente objetivo: determinar los agentes etiológicos más comunes, la respuesta antibiótica y los factores de riesgo asociados en estados de sepsis neonatal de manera específica para cada UCIN.

\section{METODOLOGÍA}

Se diseñó un estudio observacional, descriptivo, con datos obtenidos retrospectivamente; población: RN productos de parto o cesárea en la unidad materno infantil del hospital regional Teodoro Maldonado Carbo (HRTMC), en el periodo de 2 años comprendido entre enero de 2013 a diciem- bre de 2014. Para la recolección de la muestra corresponde a los pacientes registrados con código CIE 10 de sepsis neonatal, de la base de datos utilizada en la unidad hospitalaria, tras obtener el correspondiente consentimiento por parte del Departamento de Investigación del Hospital.

Los criterios de inclusión fueron: $\mathrm{RN}$ con signos clínicos de infección más hemocultivo positivo (sepsis confirmada), RN con signos clínicos de infección más dos exámenes de laboratorio con cifras alteradas; en caso de hemocultivo negativo. (sepsis probable), $\mathrm{RN}$ con signos clínicos de infección más Proteina C reactiva elevada, IL6, IL8 alteradas en caso de hemocultivo negativo (sepsis posible); mientras que los criterios de exclusión fueron: RN con signos de infección en los que no se pudo probar de manera analítica el diagnóstico de sepsis neonatal. RN transferidos de otras unidades de salud, nacidos fuera del HTMC.

Para cumplir con estos criterios se recolectaron datos analíticos en los siguientes parámetros, biometría hemática: leucocitos $<4.000 \times 10^{9} / \mathrm{L}$ ó $>34000 \times 10^{9} / \mathrm{L}$, plaquetas $<10000 \times 10^{9} / \mathrm{L}$, reactantes de fase aguda: Procalcitonina $>2.4 \mathrm{ng} / \mathrm{dl}$ después de las primeras 48 horas de vida, hemocultivo y antibiograma. Con los datos recolectados se clasificó a los casos en: sepsis confirmada y sepsis clínica: probable, posible. Todos los datos se registraron en hojas de cálculo de plantilla excel.

Para la clasificación de tipo de sepsis según su tiempo de aparición, se tomaron en cuenta tres parámetros: clínico, analítico y riesgo infeccioso materno, tomando como punto de corte las primeras 72 horas de vida. En los criterios analíticos se incluyó la positividad de hemocultivo. Para la determinante de riesgo infeccioso materno se obtuvo datos de infección de vías urinarias y/o leucorreas en la madre que no recibieron tratamiento médico o que fueron tratadas pero no curadas durante el último trimestre de la gestación. También se incluyó diagnóstico de ruptura prematura de membranas mayor a 18 horas, fiebre materna o corioamnionitis. De ser positivo el riesgo infeccioso y en concordancia con el resto de parámetros, se clasificaría como sepsis temprana., ${ }^{4,5}$

En cuanto a los factores de riesgo asociados a sepsis neonatal, se consideraron variables obstétricas y fetales. Variables obtenidas de la madre: edad de riesgo $<19$ años o $>35$ años, tipo de parto (eutócico o cesárea), riesgo infeccioso materno que comprende infecciones de vías urinarias o leucorreas 
en el último trimestre del embarazo, ruptura prematura de membranas $>18$ horas, corioamnionitis y trastornos hipertensivos del embarazo. Las variables para riesgo de origen fetal fueron: género, edad gestacional medida por escala de Ballard considerando pretérmino $<37 \mathrm{~s}$.g. y pretérmino extremo <32s.g., bajo peso al nacer $<2500 \mathrm{~g}$ y muy bajo $<1500 \mathrm{~g}$ y APGAR $<7$ en el primer minuto. Para este estudio se siguieron las recomendaciones de la Declaracion de Helsinki.

\section{RESULTADOS}

En el estudio ingresaron 163 pacientes etiquetados con códigos CIE 10 de sepsis neonatal entre los años 2012 al 2014 en la Unidad de Cuidados Intensivos Neonatales (UCIN) del HRTMC. Al aplicar los criterios de inclusión se obtuvo: 34 pacientes con sepsis confirmada, 35 pacientes con sepsis clínica, correspondiendo todos a sepsis probable. No se obtuvo diagnóstico de sepsis posible. En 94 pacientes que corresponden al $58 \%$ de la muestra, no se pudo comprobar el diagnóstico de sepsis mediante los criterios de inclusión, figura 1. La prevalencia de sepsis neonatal clínica y confirmada fue del $42 \%$. A partir de la muestra obtenida (69 casos) se clasificó sepsis en temprana o tardía; obteniéndose 28 pacientes con sepsis temprana y 41 con sepsis tardía.

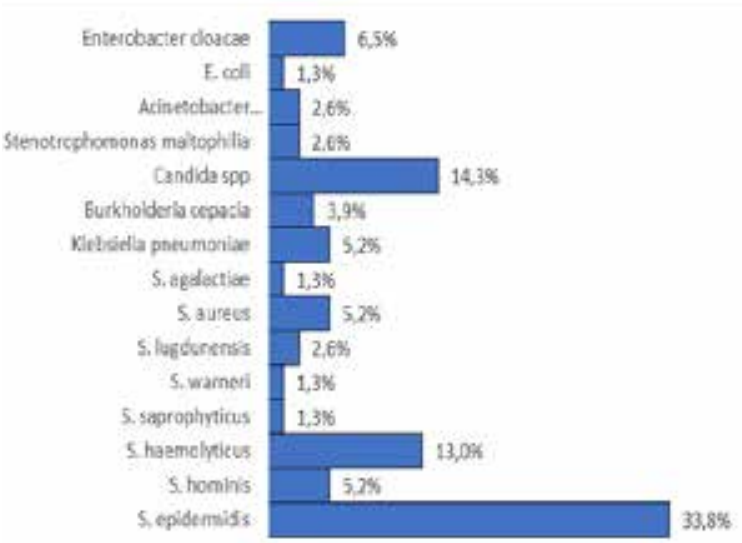

Figura 1. Frecuencia del microorganismo aislado

De los pacientes con sepsis confirmada o clínica $37 \%$ (n 25) pertenecieron al género femenino y $68 \%$ (n 43) fueron varones. La edad promedio fue de $33.26 \pm 4.52 \mathrm{~s}$.g., con un intervalo entre 32.14 y 34.39 s.g., siendo la edad máxima $42 \mathrm{s.g}$. y edad mínima 20 s.g. La moda fue $32 \mathrm{s.g}$.

En lo que corresponde a antecedentes obstétricos, $83 \%$ (n 53) de los neonatos fueron obtenidos por cesárea, mientras que $17 \%$ (n 11) fueron productos de parto eutócico; $31 \%$ (n 18) de las madres tuvieron edad de riesgo y se obtuvo una edad materna media de $29.07 \pm 6,7$ años, con un intervalo entre 27 y 30 años; la edad mínima fue de 15 años mientras que la edad máxima fue de 43 años; la moda fue 31 años.

Así mismo 20 neonatos (29\%) tuvieron APGAR menor a 7 en el primer minuto. En cuanto a los factores de riesgo fetales, la condición de pretérmino extremo <32s.g. es el único factor que resultó con riesgo significativo para sepsis tardía $(\mathrm{p}=0,017$; $\mathrm{X}^{2}=5.69$; IC 95\%).

De la muestra estudiada 69\%(n44) de los RN tuvieron riesgo infeccioso materno. $27 \%$ (n17) de las madres fueron diagnosticadas con ruptura prematura de membranas $>18$ horas, $24 \%$ (n16) presentaron trastornos hipertensivos del embarazo y $5 \%$ (n 3) corioamnionitis; esta nosología fue el único factor obstétrico que presentó valor de riesgo estadísticamente significativo para sepsis temprana. ( $\mathrm{p}=$ 0,$041 ; \mathrm{X}^{2}=0,15 ;$ IC 95\%). Tabla 1 .

TABLA 1. FACTORES DE RIESGO ASOCIADOS A SEPSIS NEONATAL SEGÚN SUTIPO

\begin{tabular}{|c|c|c|c|c|c|c|c|c|}
\hline \multirow{2}{*}{$\begin{array}{l}\text { FACTORES DE } \\
\text { RIESGO }\end{array}$} & \multicolumn{2}{|c|}{$\begin{array}{c}\text { SEPSIS } \\
\text { TEMPRANA }\end{array}$} & \multicolumn{2}{|c|}{$\begin{array}{l}\text { SEPSIS } \\
\text { TARDÍA }\end{array}$} & \multicolumn{2}{|c|}{ TOTAL } & \multirow{2}{*}{$x^{2}$} & \multirow{2}{*}{$\begin{array}{c}\text { VALOR } \\
\text { P }\end{array}$} \\
\hline & $\mathbf{F}$ & $\mathbf{P}$ & $\mathbf{F}$ & $\mathbf{P}$ & $\mathbf{F}$ & $\mathbf{P}$ & & \\
\hline \multicolumn{9}{|c|}{ FACTORES FETALES } \\
\hline $\begin{array}{l}\text { Bajo peso al } \\
\text { nacer } \\
2500-1500 \mathrm{~g}\end{array}$ & 10 & $40 \%$ & 15 & $60 \%$ & 25 & $37,31 \%$ & 0,52 & 0,81 \\
\hline $\begin{array}{l}\text { Muy bajo peso } \\
<1500 \mathrm{~g}\end{array}$ & 10 & 38,465 & 16 & $61,53 \%$ & 26 & $38,23 \%$ & 0,12 & 0,72 \\
\hline $\begin{array}{l}\text { Pretérmino } \\
37-32 \mathrm{sg}\end{array}$ & 7 & $30.43 \%$ & 8 & $69,56 \%$ & 23 & $33,3 \%$ & 1,47 & 0,22 \\
\hline $\begin{array}{l}\text { Pretérmino } \\
\text { extremo<32sg }\end{array}$ & 13 & $68.09 \%$ & 10 & $38.09 \%$ & 21 & $30,43 \%$ & 5,69 & ${ }^{*} 0,017$ \\
\hline $\begin{array}{l}\text { APGAR } \\
<7 \text {. 1er min }\end{array}$ & 10 & $50 \%$ & 24 & $50 \%$ & 20 & $29,41 \%$ & 0,91 & 0,33 \\
\hline \multicolumn{9}{|c|}{ FACTORES OBSTÉTRICOS } \\
\hline $\begin{array}{l}\text { Riesgo } \\
\text { infeccioso } \\
\text { materno }\end{array}$ & 21 & $46,66 \%$ & 24 & $53,33 \%$ & 45 & $68,18 \%$ & 1.93 & 0,16 \\
\hline $\mathrm{RPM}>18$ & 7 & $38,88 \%$ & 11 & $61.1 \%$ & 18 & $27,27 \%$ & 0,12 & 0,72 \\
\hline $\begin{array}{l}\text { Corioamnio- } \\
\text { nitis }\end{array}$ & 3 & $100 \%$ & 0 & $0 \%$ & 3 & $4,61 \%$ & 4,15 & ${ }^{*} 0,041$ \\
\hline $\begin{array}{l}\text { Trastornos } \\
\text { hipertensivos }\end{array}$ & 6 & $37.5 \%$ & 10 & $62.5 \%$ & 16 & $13,88 \%$ & 0,15 & 0,68 \\
\hline
\end{tabular}

Fuente: UCIN HTMC.

De los 69 pacientes con sepsis neonatal en el estudio, en 53 pacientes $(75,7 \%)$ se aisló algún microorganismo (bacteria u hongo) por cultivo hemático; 12 pacientes tuvieron hemocultivo negativo y a 3 pacientes no se les realizó este examen. 
Del total de las muestras cultivadas fueron positivas 53; se aisló un microorganismo en 37 cultivos (70\%); y múltiples microorganismos se aislaron en 16 muestras (30\%). De las muestras positivas se encontraron los siguientes microorganismos: estafilococo epidermidis ( $n: 26)$, candida spp $(n: 11)$, estafilococo haemolitycus ( $n: 10)$, enterobacter cloacae (n:5), Estafilococo hominis (n: 4), klebsiella pneumoniae (n:4), estafilococo aureus (n:4), burkholderia cepacia (n:3), stenotrophomona maltophilia ( $n: 2)$, acinetobacter baumanni/haemolitycus ( $\mathrm{n}: 2$ ), estafilococo lugdunensis (n:2), escherichia coli ( $\mathrm{n}: 1)$, estafilococo saprophyticus (n:1), estafilococo warneri (n:1), estreptococo agalactiae (n: 1). Figura 1.

En la tabla 2 se presenta la frecuencia de los microorganismos aislados según el tipo de sepsis, donde se obtuvo un alto porcentaje de infección por S. epidermidis en sepsis temprana, como en sepsis tardía.

\section{TABLA 2. FRECUENCIA DE MICROORGANISMO AISLADO SEGÚN TIPOS DE} SEPSIS

\begin{tabular}{lllllll}
\hline \multicolumn{3}{c}{ SEPSIS TEMPRANA } & \multicolumn{3}{c}{ SEPSIS TARDíA } \\
\hline 1 & S. epidermidis & 9 & $32 \%$ & S. Epidermidis & 17 & $35 \%$ \\
\hline 2 & Candida spp & 3 & $11 \%$ & Cándida spp & 8 & $16 \%$ \\
\hline 3 & S. haemolyticus & 3 & $11 \%$ & S. haemolyticus & 7 & $14 \%$ \\
\hline 4 & S. aureus & 3 & $11 \%$ & Enterobacter cloacae & 5 & $10 \%$ \\
\hline 5 & S. hominis & 2 & $7 \%$ & S. hominis & 2 & $4 \%$ \\
\hline 6 & $\begin{array}{l}\text { Klebsiella } \\
\text { pneumoniae }\end{array}$ & 2 & $7 \%$ & $\begin{array}{l}\text { Klebsiella } \\
\text { pneumoniae }\end{array}$ & 2 & $4 \%$ \\
\hline 7 & S. lugdunesis & 2 & $4 \%$ & Burkholderia cepacia & 2 & $4 \%$ \\
\hline 8 & S. saprophyticus & 1 & $4 \%$ & St. maltophilia & 2 & $4 \%$ \\
\hline 9 & S. agalactiae & 1 & $4 \%$ & $\begin{array}{l}\text { Acinetobacter b/ } \\
\text { haemolyticus }\end{array}$ & 2 & $4 \%$ \\
\hline 10 & E. coli & 1 & $4 \%$ & S. aureus E. coli & 1 & $2 \%$ \\
\hline 11 & Burkholderia cepacia & 1 & $4 \%$ & S. warneri & 1 & $2 \%$ \\
\hline 12 & Enterobacter cloacae & 0 & $0 \%$ & S. lugdunesis & 0 & $0 \%$ \\
\hline 13 & St. maltophilia & 0 & $0 \%$ & S. saprophyticus & 0 & $0 \%$ \\
\hline 14 & $\begin{array}{l}\text { Acinetobacter b/ } \\
\text { haemolyticus }\end{array}$ & 0 & $0 \%$ & S. agalactiae & 0 & $0 \%$ \\
\hline 15 & S. warneri & 0 & $0 \%$ & E. coli & 0 & $0 \%$
\end{tabular}

Fuente: UCIN HTMC

Los estafilococos coagulasa negativos representaron el 54\% de los hemocultivos realizados, predominando el estafilococo epidermidis; se analizó la susceptibilidad antibiótica de los mismos encontrándose lo siguiente: tabla 3 (a-b) Dentro del estudio se obtuvo un $5,4 \%$ de klebsiella pneumonie carbapenemasa positivo (KPC).
Como dato adicional del estudio, se obtuvo que las comorbilidades mayormente asociadas a sepsis neonatal fueron: neumonía con una prevalencia del $8 \%$, enterocolitis necrotizante $16 \%$ y $71 \%$ de los neonatos sépticos presentaron dificultad respiratoria.

TABLA 3A. PATRONES DE SENSIBILIDAD ANTIBIÓTICA DE LOS MICROORGANISMOS MÁS COMUNES AISLADOS

\begin{tabular}{|c|c|c|c|c|c|c|c|c|c|c|}
\hline & 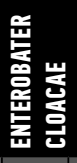 & 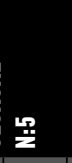 & 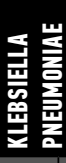 & & 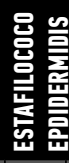 & $\frac{0}{\tilde{n}}$ & 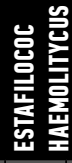 & 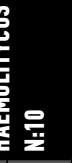 & 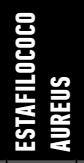 & 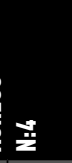 \\
\hline 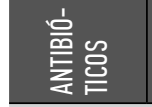 & $S$ & $R$ & $S$ & $R$ & $s$ & $R$ & $s$ & $\mathrm{R}$ & S & $R$ \\
\hline Ampicilina & $0 \%$ & $100 \%$ & $0 \%$ & $100 \%$ & $4 \%$ & $96 \%$ & $0 \%$ & $100 \%$ & $25 \%$ & $75 \%$ \\
\hline Gentamicina & $0 \%$ & $100 \%$ & $25 \%$ & $75 \%$ & $4 \%$ & $96 \%$ & $0 \%$ & $100 \%$ & $25 \%$ & $75 \%$ \\
\hline mikacina & $60 \%$ & $40 \%$ & $25 \%$ & $75 \%$ & - & - & - & - & - & - \\
\hline Meropenem & $100 \%$ & 0 & $25 \%$ & $75 \%$ & - & - & - & -- & - & - \\
\hline mipene & $100 \%$ & 0 & $25 \%$ & $75 \%$ & - & - & - & - & - & - \\
\hline Cefepime & $0 \%$ & $100 \%$ & $25 \%$ & $75 \%$ & - & - & - & - & - & - \\
\hline Ceftriaxona & $0 \%$ & $100 \%$ & $25 \%$ & $75 \%$ & $4 \%$ & $96 \%$ & $0 \%$ & $100 \%$ & $25 \%$ & $75 \%$ \\
\hline $\begin{array}{l}\text { Ciprofloxa- } \\
\text { cina }\end{array}$ & $80 \%$ & $20 \%$ & $25 \%$ & $75 \%$ & $23 \%$ & $77 \%$ & $0 \%$ & $100 \%$ & $25 \%$ & $75 \%$ \\
\hline Moxifloxacina & - & - & - & - & $100 \%$ & $0 \%$ & $100 \%$ & $0 \%$ & $100 \%$ & $0 \%$ \\
\hline Levofloxacina & $100 \%$ & $0 \%$ & $25 \%$ & $75 \%$ & $77 \%$ & $23 \%$ & $0 \%$ & $100 \%$ & $100 \%$ & $0 \%$ \\
\hline Eritromicina & - & - & - & - & $31 \%$ & $69 \%$ & $0 \%$ & $100 \%$ & $25 \%$ & $75 \%$ \\
\hline Clindamicina & - & - & - & - & $35 \%$ & $65 \%$ & $40 \%$ & $60 \%$ & $0 \%$ & $100 \%$ \\
\hline $\begin{array}{l}\text { Amoxicilina / } \\
\text { clavulánico } \\
\end{array}$ & $0 \%$ & $100 \%$ & $25 \%$ & $75 \%$ & $4 \%$ & $96 \%$ & $0 \%$ & $100 \%$ & $25 \%$ & $75 \%$ \\
\hline $\begin{array}{l}\text { Ticarcilina / } \\
\text { clavulanico } \\
\end{array}$ & $20 \%$ & $80 \%$ & $25 \%$ & $75 \%$ & - & - & - & - & - & - \\
\hline $\begin{array}{l}\text { Piperazilina } \\
\text { tozobactam }\end{array}$ & $25 \%$ & $75 \%$ & $25 \%$ & $75 \%$ & - & - & - & - & - & \\
\hline
\end{tabular}

Fuente: UCIN HTMC

TABLA 3B. PATRONES DE SENSIBILIDAD ANTIBIÓTICA DE LOS MICROORGANISMO MÁS COMUNES AISLADOS

\begin{tabular}{|c|c|c|c|c|c|c|c|c|}
\hline & 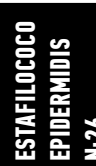 & & 啹总 & & 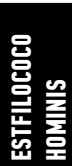 & & 兽 & \\
\hline ANTIBIÓTICOS & $S$ & P & $S$ & P & S & P & S & $\mathrm{P}$ \\
\hline Oxacilina & $4 \% \quad 9$ & $96 \%$ & $0 \%$ & $100 \%$ & $25 \%$ & $75 \%$ & $25 \%$ & $75 \%$ \\
\hline Ceftriaxona & $25 \% 7$ & $75 \%$ & $4 \%$ & $96 \%$ & $0 \%$ & $100 \%$ & $25 \%$ & $75 \%$ \\
\hline Ciprofloxacina & $25 \% \quad 7$ & $75 \%$ & $23 \%$ & $77 \%$ & $0 \%$ & $100 \%$ & $25 \%$ & $75 \%$ \\
\hline Levofloxacina & $25 \%$ & $75 \%$ & $77 \%$ & $23 \%$ & $0 \%$ & $100 \%$ & $100 \%$ & $0 \%$ \\
\hline Eritromicina & - & - & $31 \%$ & $69 \%$ & $0 \%$ & $100 \%$ & $25 \%$ & $75 \%$ \\
\hline Clindamicina & - & - & $35 \%$ & $65 \%$ & $40 \%$ & $60 \%$ & $0 \%$ & $100 \%$ \\
\hline $\begin{array}{l}\text { Amoxicilina / } \\
\text { clavulánico }\end{array}$ & $25 \% 7$ & $75 \%$ & $4 \%$ & $96 \%$ & $0 \%$ & $100 \%$ & $25 \%$ & $75 \%$ \\
\hline Vancomicina & $100 \%$ & $0 \%$ & $100 \%$ & $0 \%$ & $100 \%$ & $0 \%$ & $100 \%$ & $0 \%$ \\
\hline Linezolid & $100 \%$ & $0 \%$ & $100 \%$ & $0 \%$ & $100 \%$ & $0 \%$ & $100 \%$ & $0 \%$ \\
\hline Moxifloxacino & - & - & $100 \%$ & $0 \%$ & $100 \%$ & $100 \%$ & $100 \%$ & $0 \%$ \\
\hline
\end{tabular}

Fuente: UCIN HTMC 
En el año 2014 se tuvo un total de 2172 nacimientos en la unidad materno infantil del HRTMC. La prevalencia de sepsis neonatal fue de 1.56 por cada 100 recién nacidos para dicho periodo. La tasa de mortalidad específica fue de 3,22 por cada 1000 recién nacidos. Como limitante para este estudio, no se contó con datos estadísticos de nacimientos y defunciones del año 2013, por lo que los datos epidemiológicos corresponden solo al periodo 2014.

De los 69 casos de sepsis neonatal confirmada o probable, fallecieron 15 pacientes, lo que refleja una letalidad del $27.73 \%$ en el periodo de 2 años 2013-2014.

DISCUSIÓN

El estudio refleja estafilococos coagulasa negativos como agentes etiológicos de sepsis neonatal, a la cabeza los S. epidermidis, seguidos por cándidaspp $(14,3 \%), S$. aureus $(5,2 \%)$, enterobactercloacae $(5,2 \%)$ y klebsiella pneumoniae $(5.2 \%)$. La prevalencia de $S$. epidermidis obtenida sostiene la hipótesis planteada para este estudio y es similar a otros realizados a nivel mundial. ${ }^{13-17}$

La alta prevalencia de S. epidermidis no solo en sepsis tardía sino también en temprana, contradice la epidemiologia clásica; en sepsis de inicio precoz son SCB y gram negativos entéricos los que comúnmente colonizan el tracto genital femenino. Sin embargo, es notable la baja prevalencia de bacterias colonizadoras del canal del parto como agentes causales de sepsis precoz en esta investigación, E. coli y S. agalactiae solo se presentaron en 2 de los casos en el presente trabajo. ${ }^{18}$

Un estudio de 116 casos realizado en China, encontró similar prevalencia; población demográficamente muy distinta a la nuestra, coincide con los resultados obtenidos en este estudio. ${ }^{19}$ Shane y colaboradores en el 2014 indican que dado el impacto de las campañas sobre profilaxis antibiótica y la disminución de sepsis neonatal por S. agalactie, la sepsis temprana tiene ahora como principales agentes causales otros patógenos gram negativos como: klebsiella pneumoniae, enterobacter cloacae, acinetobacter y streptomona maltophila, premisa que se cumple en este estudio. ${ }^{9}$

Se cree que la baja prevalencia de partos en la población estudiada, disminuye aun más el riesgo de infección por S. agalactiae y E. coli, ya que los RN evitan su paso por el canal del parto al ser obtenidos por cesárea que tuvo una prevalencia del 83\%. La prevalencia de infecciones ascendentes prenatales es baja en la muestra obtenida; sólo el $5 \%$ de las gestantes presentaron corioamnionitis y $27 \%$ RPM; se debe al uso de antibióticos profilácticos estandarizado. ${ }^{22,23}$

La infección fúngica se relaciona con la estancia hospitalaria prolongada, por lo que algunos estudios sugieren el uso de fluconazol profiláctico en RN de muy bajo peso ( $<1000 \mathrm{~g}$ ), durante las 6 primeras semanas de vida si es que la unidad de cuidados en cuestión tiene altas tasas de infección fúngica (nivel de evidencia $\mathrm{AI}$ ); como es el caso de la UCIN estudiada. .,5,9

En RN pretérmino, población que fue prevalente (76\% de la muestra) en el presente estudio, tiene mayor riesgo de padecer esta patología. .,24 $^{2}$

En la clasificación de sepsis clínica no se obtuvo ningún paciente con sepsis posible ya que para cumplir con el criterio, se requieren los valores de PCR, análisis con el que no siempre se cuenta; por ello se decidió aplicar los conceptos de sepsis clínica para esta investigación. 4,5,7,20

El tipo de sepsis tardía es predominante con el 59\% frente al $41 \%$ de tipo temprana. Es posible que este dato se debe a la profilaxis antibiótica instaurada contra el SGB. En un estudio de casos y controles se refleja, que en $\mathrm{RN}$ que desarrollaron sepsis tardía grave, estuvieron mayormente expuestos a antibiótico terapia intraparto, demostrando en la población de pacientes infectados, mayor resistencia a la ampicilina ${ }^{25}$.

Existe una mayor prevalencia e incidencia de sepsis de tipo tardio a nivel mundial ${ }^{11,16,18 ~ " T h e ~}$ Journal of Pediatrics", 2015, 452 episodios de sepsis; en el que $90 \%$ de los casos fueron de presentación tardía, tomando como punto de corte para la clasificación, 72 horas de vida. ${ }^{13}$ Para citar una fuente socio-demográficamente más similar a nuestra población, Perú reporta que $30.8 \%$ de los casos presentaron sepsis precoz, y $69,2 \%$ presentó sepsis tardia. ${ }^{14}$ similar epidemiología tiene la UCIN del HTMC.

En cuanto a los antibiogramas de manera alarmante, el 75\% de cepas de klebsiella pneumoniae obtenidas, presentan resistencia a la ampicilina; iguales resultados se obtienen para imipenem, ticarcilina y piperacilina, lo que refleja una emergente población de klebsiella pneumoniae productora de carbapenemasas (KPC 5,6\%). 
CONCLUSIÓN

Dado que S. epidermidis fue el patógeno prevalente en sepsis neonatal temprana y tardía, se concluye que la microbiología esperada en sepsis temprana se ve alterada por el uso profiláctico de antibióticos frente al riesgo de infección materna y a la baja prevalencia de parto en la población estudiada. El control de los factores de riesgo obstétricos reduce la incidencia de sepsis neonatal por esta causa; sin embargo, surgen de manera concomitante las infecciones por microorganismos nosocomiales, sobre todo el RN pretérmino.

La incidencia de sepsis por estafilococos coagulasa negativos es creciente. Frente a esto es vital el cumplimiento estricto de los protocolos de higiene y seguridad del personal; algunos artículos recomiendan también la adición de antibióticos tipo vancomicina para los procesos invasivos. ${ }^{21}$

De los casos con sospecha de sepsis en la UCIN HRTMC, el 58\% de los neonatos fueron tratados con antibioticoterapia de amplio espectro sin presentar sepsis neonatal de manera probada. El uso indiscriminado de antibióticos de amplio espectro, aumenta los niveles de resistencia antibiótica, incrementa el riesgo de infecciones por patógenos oportunistas y genera una creciente población de bacterias multidrogo resistentes y por tanto aumenta la mortalidad. Es por ello que se recomienda el uso mesurado de estos tratamientos ante la sospecha de sepsis neonatal.

\section{REFERENCIAS BIBLIOGRÁFICAS}

1. Polin Richard, MD and the COMMITEE ON FETUS AND NEWBORN. Management o Neonates with Suspected or Proven Early-Onset Bacterial Sepsis. American Academy of Pediatrics; 2012; 129 (5): 1006. Disponible en: http://pediatrics.aappublications.org/ content $/ 142 / 3$ ?current-issue $=y$

2. Asociación Española de Pediatría. España: Colomer B, Coto Cotallo D, Ramos Aparicio A, Ibañez Fernández A. [2008, 2016]. AEP Asociación Española de Pediatría; 189. Disponible en: https://www.aeped.es/protocolos/

3. Gaitán SC, Camacho MG. Sepsis Neonatal. Precop scp ccap. 2012; 13 (2): 7-26

4. Andres CG, Paul WS, Barbara JS. Neonatal Infectious Diseases: Evaluation of Neonatal Sepsis. Pediatr Clin North Am. 2013; 60(2): 367-389. Disponible en: https://www.ncbi.nlm.nih.gov/pmc/articles/ PMC4405627/

5. Coronel W, Pérez C, Guerrero C, Bustamante H. Sepsis Neonatal. Revista de Enfermedades Infecciosas en Pediatría. 2009. ;23(90): 57-68
6. Galvis Díaz E. Guía Clinica Latinoamericana de Cuidados Neonatales: Diagnóstico de infección en el recién nacido. Curso Latinoamericano de Cuidados Neonatales; 2009.

7. Zea A, Turin C. Unificar los Criterios de Sepsis Neonatal Tardía: Propuesta de un Algoritmo de Vigilancia Diagnóstica. Rev Peru Med Exp Salud Publica. 2014; 31(2):358-363. Disponible en: http://www.researchgate.net/publication/264162582

8. United Nations Children's Fund [Internet].Estados Unidos: You Danzhen, Hug Lucia,Yao Chen. 2014. Child Mortality; 1-29. Disponible en: http://www. childmortality.org/files_v21/download/unicef2013-child-mortality-report-LR-10_31_14_195.pdf

9. Shane Al, Stoll Bj. Neonatal Sepsis: Progress Towards Improved Outcomes. J Infect. 2014; 68 (1): 24-32.

10. Oza Shefali, Lawn Joy E, Hogan Daniel R, Mathers Colin \&Cousens Simon. Neonatal Cause of Death Estimates for the Early and Late Neonatal Periods for 194 countries: 2000-2013. Bulletin of the World Health Organization. 2014; 93(1): 19-28.

11. Donoso A, Arriaga D, Cruces P, Díaz F. Shock Séptico en Pediatría Enfoque en el Diagnóstico y Tratamiento. Rev Chil Pediatr. 2013; 84 (5): 484-498

12. Instituto Nacional de estadísticas y censos [Internet]. Ecuador: Usiña J, Carrera S. 2013. Ecuador en cifras; 182-187. Disponible en: http://www.ecuadorencifras. gob.ec/documentos/web-inec/Poblacion_y_Demografia/Nacimientos_Defunciones/Publicaciones/ Anuario_Nacimientos_y_Defunciones_2013.pdf

13. Matthew J. Bizzarro, VeronikaShabanova y col. Neonatal Sepsis 2004-2013: The Rise and Fall of CoagulaseNegative Staphylococci. J Pediatr. 2015; 166(5):1193-9.

14. Biblioteca Digital. Oficina de sistemas e informática UNT [Internet]. Perú: González Saravia, Iris Liliana. Agentes etiológicos de sepsis neonatal bacteriana y patrón de sensibilidad antimicrobiana en el servicio de neonatología del Hospital IV Víctor Lazarte Echegaray 2007-2012; 2013 [2014-10-14]. Disponibleen: http:// dspace.unitru.edu.pe/handle/UNITRU/269?show=full

15. José TI, Cristina RT, M. Isabel SR, Fernando AC, $M$. Eugenia PA, Álvaro GM y col. Sepsis neonatal en la era de profilaxis antimicrobiana prenatal. Rev Chil Infect 2007; 24 (2): 111-116

16. Salvador SG, Ismael SG. Cultivos positivos y su relación con sepsis neonatal en la Unidad de Cuidados Intensivos Hospital de Guadalajara. Hipoc Rev Med. 2011; 24:05-11

17. Genes L, Lacarrubba J, Mir R, Céspedes E, Mendieta E. Sepsis neonatal. Caracterización en Recién Nacidos de muy bajo peso. Experiencia de once años. Pediatr. (Asunción). 2013; 40(2): 145 - 1542013

18. Lona J, Verdug M, Pérez R, Pérez J, Ascencio E, Benítez E. Etiología y patrones de resistencia antimicrobiana en sepsis neonatal temprana y tardía, en una Unidad 
de Terapia Intensiva Neonatal. Arch Argent Pediatr. 2015; 113(4):317-323.

19. Zhiling Li, ZhijunXiao, Zhiping Li, QiaoZhong, Ye Zhang, FengXu. 116 cases of neonatal early-onset or late-onset sepsis: A single center retrospective analysis on pathogenic bacteria species distribution and antimicrobial susceptibility. Int Journal Clinic Experience Med. 2013; Vol 6(8):693-699.

20. Haque, Khalid N. Definitions of bloodstream infection in the newborn. Pediatric Critical Care Medicine. 2005; Vol 6 (3): 45-49.

21. Marchant E, Boyce G, Sadarangani M, Lavoie P. Neonatal Sepsis due to Coagulase-Negative Staphylococci. Clin Dev Immunol. 2013; 2013: 1-10.
22. Centers for Disease Control and Prevention, Prevention of Perinatal Group B Streptococcal Disease. Morbility and Mortality Weekly Report. 2010; 59(RR10): 3-6.

23. Ministerio de Salud Pública. Sepsis neonatal. Guía de Práctica Clínica. Primera edición. Quito: MSP; 2015.

24. Goldstein B, Giroir B, Randolph A, and the Members of the International Consensus Conference on Pediatric Sepsis. International pediatric sepsis consensus conference: Definitions for sepsis and organ dysfunction in pediatrics. Pediatric Critic CareMed. 2005; 6 (1): 2-8

25. Glasgow TS1, Young PC, Wallin J, Kwok C, Stoddard G, Firth S, y col. Association of intrapartum antibiotic exposure and late-onset serious bacterial infections in infants. Pediatrics. 2005; 116(3):696-702. 